\title{
Pollutant Concentrations in Street Canyons of Different Aspect Ratio with Avenues of Trees for Various Wind Directions
}

\author{
Christof Gromke • Bodo Ruck
}

Received: 23 March 2010 / Accepted: 24 January 2012 / Published online: 11 February 2012

(C) Springer Science+Business Media B.V. 2012

\begin{abstract}
This study summarizes the effects of avenues of trees in urban street canyons on traffic pollutant dispersion. We describe various wind-tunnel experiments with different tree-avenue models in combination with variations in street-canyon aspect ratio $\mathrm{W} / \mathrm{H}$ (with $W$ the street-canyon width and $H$ the building height) and approaching wind direction. Compared to tree-free street canyons, in general, higher pollutant concentrations are found. Avenues of trees do not suppress canyon vortices, although the air ventilation in canyons is hindered significantly. For a perpendicular wind direction, increases in wall-average and wall-maximum concentrations at the leeward canyon wall and decreases in wall-average concentrations at the windward wall are found. For oblique and perpendicular wind directions, increases at both canyon walls are obtained. The strongest effects of avenues of trees on traffic pollutant dispersion are observed for oblique wind directions for which also the largest concentrations at the canyon walls are found. Thus, the prevailing assumption that attributes the most harmful dispersion conditions to a perpendicular wind direction does not hold for street canyons with avenues of trees. Furthermore, following dimensional analysis, an estimate of the normalized wall-maximum traffic pollutant concentration in street canyons with avenues of trees is derived.
\end{abstract}

Keywords Air quality - Maximum concentration · Pollutant dispersion · Street canyon · Tree-avenue · Urban Vegetation

C. Gromke $(\varangle)$

Building Physics and Systems, Eindhoven University of Technology, Den Dolech 2, 5612 AZ Eindhoven, The Netherlands

e-mail: c.gromke@gmx.net

C. Gromke

WSL Institute for Snow and Avalanche Research SLF, Flueelastr. 11, 7260 Davos, Switzerland

C. Gromke · B. Ruck

Institute for Hydromechanics, Karlsruhe Institute of Technology KIT, Kaiserstrasse 12, 76128 Karlsruhe, Germany 


\section{Introduction}

Traffic emissions are a major source of air pollution in urban areas. Critical and harmful situations often arise in densely built-up inner city areas with street canyons suffering from limited ambient air exchange and high pollutant concentrations. Trees inside street canyons can even accentuate the problem since they affect flow and pollutant transport. In particular, in urban street canyons with avenues of trees, where the lower and the upper canyon areas are separated by a layer of tree crowns, the air exchange with the above-roof flow is hindered and traffic emissions released at the street level may become trapped.

The current state of knowledge encompasses various aspects of air quality in tree-free street canyons. Mean flow and turbulence, as well as pollutant dispersion and concentrations, partly including thermal effects, traffic-induced turbulence and chemical reactions of air constituents have been investigated in field studies (e.g. DePaul and Sheih 1985, 1986; Schatzmann and Leitl 2002; Eliasson et al. 2006), laboratory wind-tunnel or water-channel experiments (e.g. Meroney et al. 1996; Kastner-Klein and Plate 1999; Pavageau and Schatzmann 1999; Baik et al. 2000; Kastner-Klein et al. 2001; Dezso-Weidinger et al. 2003) and numerical simulations (e.g. Sini et al. 1996; Baik and Kim 1999, 2002; Liu and Barth 2002; So et al. 2005; Baik et al. 2007; Moonen et al. 2011a). Moreover, state-of-the-art summaries of streetcanyon-dispersion modelling are provided in Vardoulakis et al. (2003), Ahmad et al. (2005) and $\mathrm{Li}$ et al. (2006). In the great majority of all such studies, however, only the flow close to perpendicular to the canyon axis has been considered, since this direction is generally supposed to yield the highest pollutant concentrations.

In contrast, flow and pollutant dispersion in street canyons with trees have been investigated by only a few authors. Gross (1997) numerically simulated the impact of avenues of trees with a microscale computational fluid dynamics (CFD) model applying a $k-\varepsilon$ turbulence model to close the steady-state Reynolds-averaged Navier-Stokes (RANS) equations. Compared to the tree-free situation, Gross reported generally lower flow velocities in the street canyon and higher traffic pollutant concentrations at the pedestrian level in front of the leeward canyon wall. Similar results were found in the RANS-based CFD simulations with $k-\varepsilon$ turbulence closure by Ries and Eichhorn (2001). Their simulations revealed pollution concentration increases close to the leeward canyon wall together with flow-velocity decreases in the presence of trees. Directly above the traffic lanes, and in-between the tree rows, increased velocities and decreased concentrations were found. It has to be noted that Gross (1997) and Ries and Eichhorn (2001) performed two-dimensional (2D) simulations that cannot completely reflect the flow topology and dispersion processes in the entire street canyon. Strictly, their results are limited to the inner area of rather long canyons where, indeed, a quasi-2D-dominated flow field exists.

Wind-tunnel investigations of flow and traffic pollutant concentrations in an isolated street canyon with avenues of trees were performed by Gromke and Ruck (2007). Tree models were placed along the axis of a canyon with street-length to building-height ratio $L / H=10$ and street-width to building-height ratio (aspect ratio) $W / H=1$. Different tree characteristics and tree-avenue configurations were studied (crown diameter, tree height, tree spacing). For a perpendicular wind direction, increased concentrations at the leeward wall and comparatively less decreased concentrations at the windward wall were measured. The tree induced concentration changes were found to be more pronounced with a larger crown diameter and smaller tree spacing, i.e. when the canyon was occupied by a greater volume of vegetation. However, the characteristic pollutant distributions with higher concentrations at the leeward walls still indicated a flow field dominated by a canyon vortex as in the tree-free situation. Gromke and Ruck (2009a) studied the effects of traffic-induced turbulence and advection on 
pollutant concentrations in the street-canyon/tree-avenue configurations of Gromke and Ruck (2007). They observed that two-way traffic led to a more homogeneous pollutant distribution with a reduction in the peak concentrations in the canyon inner area. The implications of traffic-induced turbulence and advection on pollutant dispersion were confined to the street-level region, in particular to the region below the tree crowns. With an increasing street-canyon volume occupied by vegetation, a strengthened channelling effect of the air affected by the traffic motion was found.

Wind-tunnel studies focusing on the sensitivity of flow and traffic pollutant dispersion to crown porosity in a street canyon with $L / H=10$ and $W / H=1$ are presented in Gromke and Ruck (2009b). For a perpendicular flow, the measurements revealed higher traffic pollutant concentrations at the leeward and lower concentrations at the windward wall with decreasing porosity. A key result is that only in the high porosity range does a distinct sensitivity of the concentrations to the crown porosity exists. In addition, velocity measurements confirmed the existence of a canyon vortex in the presence of avenues of trees, however, with lower flow speed compared to the tree-free situation. These wind-tunnel experiments were the basis for CFD steady-state RANS simulations (Gromke et al. 2008; Balczó et al. 2009) and largeeddy simulations (LES) (Salim et al. 2011; Moonen et al. 2011b). The RANS simulations employing $k-\varepsilon$ turbulence closure and a Reynolds-stress model (RSM) generally underestimated the flow velocities and overestimated the pollutant concentrations when the turbulent Schmidt number default values were used. A turbulent Schmidt number $S c_{\mathrm{t}}$ parameter study in Gromke et al. (2008) showed best agreement to experimental concentration data for $S c_{\mathrm{t}}=$ $0.2-0.5$ for the $k-\varepsilon$ turbulence model and for $S c_{\mathrm{t}}=0.5-0.6$ for the RSM. However, these optimum $S c_{\mathrm{t}}$ values are restricted to the street canyon under investigation, since they depend strongly on the geometry, the flow topology and the pollutant source position (Blocken et al. 2008). The more recent studies of Salim et al. (2011) and Moonen et al. (2011b) showed that LES is capable of providing reliable flow and concentration data in better agreement with the wind-tunnel measurements than the RANS simulations. However, compared to RANS simulations, extensive computational costs are involved for LES.

The present authors further performed wind-tunnel dispersion studies in street canyons of different aspect ratios and with various tree-avenue configurations subjected to different wind directions. For a perpendicular, oblique and parallel wind direction, street canyons $(L / H=10)$ with aspect ratio of $W / H=1$ and 2 with one or two rows of avenues of trees were investigated. The crown porosity and tree-stand density of the avenues of trees were varied. The concentration data of these wind-tunnel experiments, together with those of Gromke and Ruck (2009b), are compiled in the internet database CODASC - Concentration Data of Street Canyons, www.codasc.de (CODASC 2008). The purpose of the database is to provide wind-tunnel concentration data for validation purposes in numerical pollutant dispersion modelling. Selected street-canyon/tree-avenue configurations from the database were numerically simulated by Buccolieri et al. (2009, 2011). Employing a Reynolds-stress turbulence model, street canyons with $W / H=2$ were investigated by Buccolieri et al. (2009) for a perpendicular flow direction and by Buccolieri et al. (2011) for an oblique flow direction with $\alpha=45^{\circ}$. Contrary to the simulations of street canyons with $W / H=1$, the CFD results generally underestimated the wind-tunnel concentrations for both wind directions. Overall, the CFD results for a perpendicular flow were in better agreement with the wind-tunnel data than for an oblique flow. Buccolieri et al. (2011) also studied the influence of avenues of trees on flow and dispersion for a real urban setting in Bari/Italy. By simulating the real and a fictitious tree-free scenario, and comparing the results with measurements, they underlined the importance of accounting for vegetation in CFD modelling for reliable urban dispersion studies. 
An unresolved issue is the potential of avenues of trees in urban-street canyons to filter out particulate matter $\left(\mathrm{PM}_{10}\right)$. Langner et al. (2011) emphasised the large potential of vegetation in street canyons to filter out $\mathrm{PM}_{10}$ relative to other urban surfaces and sites. However, a reliable quantification of the effective filtering performance remains difficult since large uncertainties in the deposition velocity and resuspension fraction of $\mathrm{PM}_{10}$ exist (e.g. Litschke and Kuttler 2008). The latter authors estimated that typical tree-avenue configurations in urban-street canyons have the potential to filter out only a few percent of the traffic-emitted $\mathrm{PM}_{10}$.

The present article provides a summary of wind-tunnel dispersion studies for 28 streetcanyon/tree-avenue configurations that can be found in the internet database CODASC (CODASC 2008). The influence and relative importance of different tree-avenue and streetcanyon characteristics on general flow and dispersion processes are discussed. In particular, the dependency of traffic pollutant concentrations on the crown porosity and tree-stand density in combination with the approaching wind direction and street-canyon aspect ratio is systematically analyzed. The article differs from the earlier publications mentioned above in that it presents a synoptic overview of the interdependencies between the investigated parameters rather than to provide detailed analyses of flow and dispersion in single streetcanyon/tree-avenue configurations. Section 2 details the wind-tunnel set-up and introduces the vegetation modelling concept employed for the tree-avenue models. In Sect. 3, the windtunnel results are presented and discussed, and based on dimensional analysis, an estimate of the wall-maximum traffic pollutant concentrations is derived in Sect. 4. A summary and the conclusions are given in Sect. 5.

\section{Methods}

\subsection{Street-Canyon Representation and the Measurement Technique}

Street-canyon representations (scale $M=1: 150$ ) were formed by two parallel aligned blocks representing full-scale dimensions for street length $L=180 \mathrm{~m}$, building height $H=18 \mathrm{~m}$ and building widths $W_{\mathrm{A}}=W_{\mathrm{B}}=18 \mathrm{~m}$. Two aspect ratios $W / H=1$ and 2 were realized, herein referred to as narrow and broad street canyons, respectively. Tree models with different crown porosity/permeability were placed along the street in different planting densities forming one or two rows of avenues of trees (Fig. 1). The release of traffic emissions was simulated by means of a tracer gas (sulphur hexafluoride $\mathrm{SF}_{6}$ ) that was emitted from line sources embedded at street level, as described in Meroney et al. (1996). It must be noted that the line sources extend beyond the street-canyon ends by $0.92 H$ on each side (Fig. 1). This arrangement accounts also for the emission of traffic exhausts on the neighbouring crossings
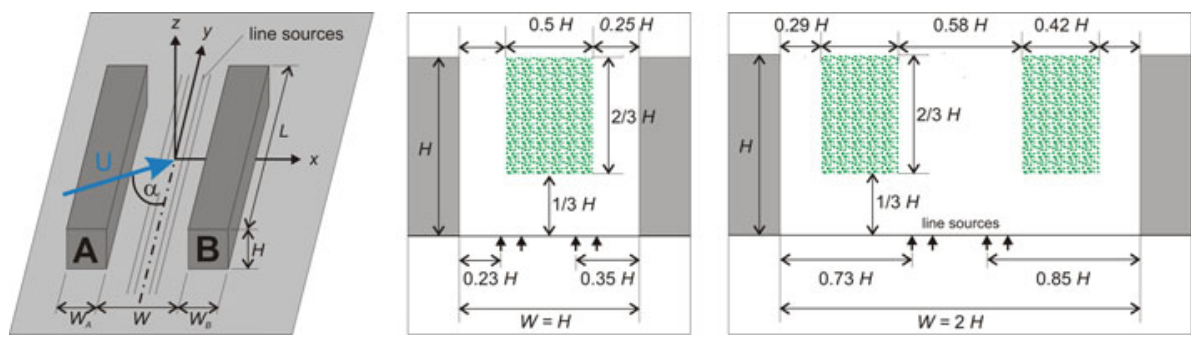

Fig. 1 Street-canyon geometry with tree-avenue arrangement $\left(W_{\mathrm{A}}=W_{\mathrm{B}}=H\right)$ 
and creates a realistic emission-source situation. It has significant influence on the pollutant concentrations in the canyon sideward areas since the traffic-exhaust releases on the crossings are transported by different flow and vortex regimes into the street canyon. Measurement taps were applied along the canyon walls $\mathrm{A}$ and $\mathrm{B}$, cylindrical tubes of diameter $d_{\mathrm{t}}=1 \mathrm{~mm}$ protruding $l_{\mathrm{t}}=5 \mathrm{~mm}(x / H=0.042)$ out of the wall, sampling the near-façade canyon air. A noticeable disturbance effect of the measurement taps on the flow was excluded for the present flow conditions. The Reynolds number $R e_{\mathrm{t}}=d_{\mathrm{t}} u_{\mathrm{t}} / v$ of the cylindrical tubes, where $u_{\mathrm{t}}$ is the flow speed at distance $l_{\mathrm{t}}$ from the wall, was estimated at $R e_{\mathrm{t}} \approx 50\left(u_{\mathrm{t}}<1 \mathrm{~m} \mathrm{~s}^{-1}\right.$, see Gromke and Ruck 2009b). For this Reynolds number the wake is still laminar (e.g. Schlichting and Gersten 2003). The concentration samples were analyzed using electron capture detection (ECD) (model: Meltron LH 108) and the concentration normalized according to

$$
c^{+}=\frac{c u_{\mathrm{H}} H}{Q_{1}}
$$

where $c^{+}$is the normalized concentration, $c$ is the measured concentration, $u_{\mathrm{H}}$ is the freestream velocity at building height $H$, and $Q_{1}$ is the tracer-gas source strength per unit length.

In the wind tunnel, an atmospheric boundary-layer flow was simulated with a power-law exponent $\alpha_{U}=0.30$ for the vertical profile of mean horizontal velocity $U(z)$ and $\alpha_{I}=-0.36$ for the turbulence intensity $I(z)$ according to

$$
\frac{\Phi(z)}{\Phi\left(z_{\mathrm{ref}}\right)}=\left(\frac{z}{z_{\mathrm{ref}}}\right)^{\alpha_{\Phi}}
$$

where $\Phi$ either is $U$ or $I$ and $z_{\text {ref }}$ is the reference height. According to the Verein Deutscher Ingenieure (VDI) guideline for environmental meteorology (VDI 3783-12 2000), both profile exponents correspond to typical atmospheric boundary-layer profiles over urban/suburban terrain. The power-law formulation given by Eq. 2 is widely accepted for the mean velocity profile in building and environmental aerodynamics wind-tunnel investigations. It is preferred to the logarithmic law-of-the-wall because it provides a description of the entire atmospheric boundary layer and not only of the surface layer. Moreover, it is more convenient from a practical point of view since the logarithmic-law parameters, namely the friction velocity, the displacement height and the aerodynamic roughness length, are often not known.

The streamwise pressure gradient was forced to zero by adjusting the wind-tunnel ceiling when the street-canyon model was set up in the test section. A more detailed description of the simulated boundary-layer flow can be found in Gromke and Ruck (2005) and in Gromke (2008). For the present investigation, a flow velocity of $U\left(z_{\text {ref }}=H\right)=4.65 \mathrm{~m} \mathrm{~s}^{-1}$ was realized. The Reynolds number $R e_{\mathrm{H}}=37,000$, based on the building height $H$ and the velocity $u_{\mathrm{H}}$, ensures a Reynolds-number independent flow and dispersion field (e.g. Snyder 1972; Meroney 2004).

\subsection{Tree Models and the Similarity Criterion}

Tree crowns are porous objects and permeable to the airflow. Flows past porous objects differ crucially from flows past non-porous objects, and characteristic differences can be found in the drag and in the wakes. Due to large volume specific surfaces of porous objects, skin friction becomes important, and it can no longer be neglected as with non-porous objects, where the pressure difference on the windward and leeward side dominates the drag. The overall drag of porous objects is approximately between 2 and 3 times larger than those of non-porous counterpart objects (Gromke and Ruck 2008). Moreover, as the tree crowns, made up of branches and leaves, are flexible and deform towards a more streamlined shape 

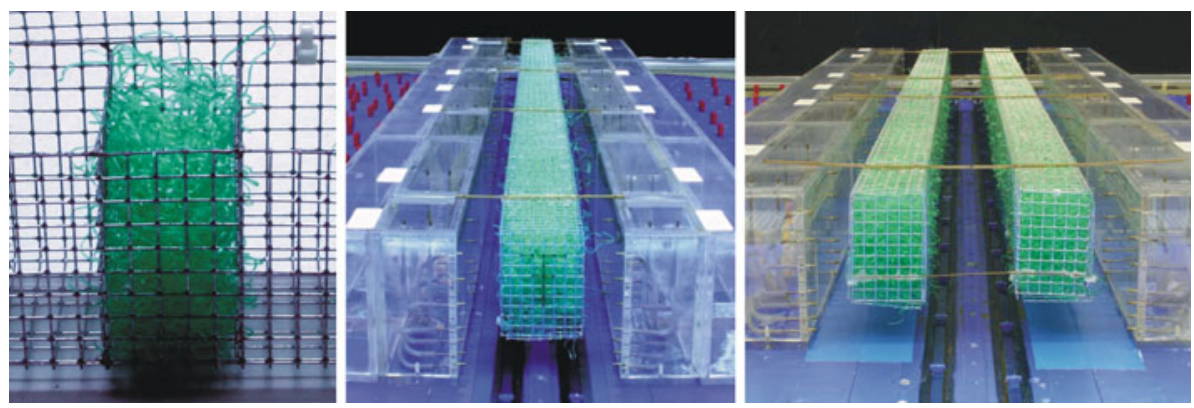

Fig. 2 Fibrous wadding material in the cage cell; street-canyon models with tree-avenues

for increasing wind speeds, a decrease in the drag coefficient with increasing wind speed is observed. Wakes of porous objects extend further downstream and the leeward recirculation zone is often detached. Gromke and Ruck (2008) investigated the flow past isolated porous and non-porous tree models with spherical crowns in a boundary-layer wind tunnel. At a downstream distance of five times the crown diameter, the mean flow behind the non-porous tree model had returned to the undisturbed approaching flow conditions, but was still highly disturbed behind the porous tree models. Contrary to the non-porous model, the wakes of the porous models showed detached regions of increased Reynolds stress $\left(\left\langle u^{\prime} w^{\prime}\right\rangle\right)$ and turbulence kinetic energy. In summary: due to their porosity, tree crowns show different aerodynamic properties in comparison to non-porous bodies that have to be accounted for in wind-tunnel investigations.

For the present study, trees were modelled using custom-made lattice-cage constructions with cross-sections of either $0.42 \mathrm{H}$ or $0.5 \mathrm{H}$ width and $0.67 \mathrm{H}$ height (Figs. 1, 2), see also Gromke (2011). The lattice cages were aligned symmetrically along the street axis representing either one or two rows of avenues of trees in the narrow $(W / H=1)$ or broad $(W / H=2)$ street canyons, respectively. Spanning the street canyon of length $L$, the cages were divided into 31 cells of $0.32 \mathrm{H}$ depth each. A fibrous synthetic wadding material was used to fill the cells, whose purpose was to facilitate a uniform distribution of the wadding material throughout the entire length of the lattice cage (Fig. 2). Different crown porosities/permeabilities were realized by homogeneously packing the cells with defined masses of the wadding material. Pore volume fractions of $P_{\mathrm{Vol}}=97.5 \%$ (herein referred to as high crown porosity) and $P_{\mathrm{Vol}}=96.0 \%$ (herein referred to as low crown porosity), typical for crown porosities of deciduous trees (Gross 1987; Ruck and Schmidt 1986), were simulated.

However, the aerodynamic characteristics of the tree models are not sufficiently and definitely determined by their crown porosities. The internal crown structure, i.e. the pore-size distribution, the arrangement and form of the crown constituting material and its surface properties are also important. In order to account for all these factors, the pressure loss coefficient $\lambda\left(\mathrm{m}^{-1}\right)$ was determined for the packed wadding material samples with pore-volume fractions of $P_{\mathrm{Vol}}=97.5 \%$ and $P_{\mathrm{Vol}}=96.0 \%$ in forced-convection conditions, according to

$$
\lambda=\frac{\Delta p_{\mathrm{st}}}{p_{\mathrm{dyn}} d}=\frac{p_{\mathrm{ww}}-p_{\mathrm{lw}}}{(1 / 2) \rho u^{2} d}
$$

where $\Delta p_{\text {st }}$ is the difference in static pressure windward (ww) and leeward (lw) of the porous sample in forced-convection conditions, $p_{\text {dyn }}$ is the dynamic pressure, $u$ is the flow speed, and $d$ is the porous sample thickness in the streamwise direction. 


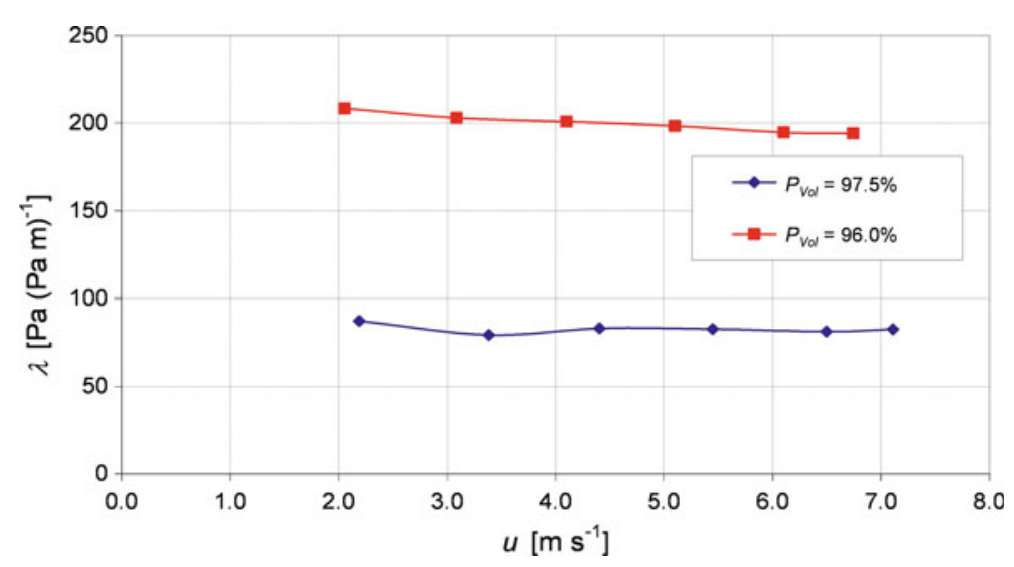

Fig. 3 Pressure loss coefficients $\lambda$ of porous samples made of fibrous wadding material

Figure 3 shows the measured pressure loss coefficients $\lambda$ for different flow speeds, where no pronounced dependency of $\lambda$ on $u$ is visible for both porosities. The slight decreases in $\lambda$ with increasing $u$ for $P_{\mathrm{Vol}}=96.0 \%$ is attributed to viscous effects. A deformation or compaction of the wadding material samples was not observed in the tested flow speed range. From these measurements, the pressure loss coefficients were determined to be $\lambda=80 \mathrm{~m}^{-1}$ and $\lambda=200 \mathrm{~m}^{-1}$ for the tree model crowns of high $\left(P_{\mathrm{Vol}}=97.5 \%\right)$ and low $\left(P_{\mathrm{Vol}}=96.0 \%\right)$ porosity, respectively.

Finally, the modelling approach still requires to be substantiated by similarity considerations providing a methodical basis for the transfer of wind-tunnel model-scale results to full-scale. The derivation of a fluid dynamical similarity criterion is based on energy considerations and expressed by the postulation that the normalized pressure losses (normalized by the dynamic pressure $p_{\mathrm{dyn}}$ ) have to be equal in full-scale (fs) and model-scale (ms), i.e.

$$
\left[\frac{\Delta p}{p_{\mathrm{dyn}}}\right]_{\mathrm{fs}}=\left[\frac{\Delta p}{p_{\mathrm{dyn}}}\right]_{\mathrm{ms}},
$$

which, with Eq. 3, yields

$$
\frac{\lambda_{\mathrm{fs}}}{\lambda_{\mathrm{ms}}}=\frac{d_{\mathrm{ms}}}{d_{\mathrm{fs}}}=M
$$

That is, the ratio of the pressure loss coefficients has to be equal to the wind-tunnel modelscale factor $M$. Applying Eq. 5 to the tree models results in $M=1: 150$, corresponding to full-scale pressure loss coefficients $\lambda_{\mathrm{fs}}=0.53 \mathrm{~m}^{-1}$ and $\lambda_{\mathrm{fs}}=1.33 \mathrm{~m}^{-1}$ for the high and low porosity, respectively.

Grunert et al. (1984) performed forced-convection experiments with sections of vegetation shelterbelts in a wind tunnel for flow speeds $u=4-11 \mathrm{~m} \mathrm{~s}^{-1}$, where the shelterbelt sections were made of broad-leaved tree branches assembled in three levels of branch density $\rho_{\mathrm{sb}}$. For low flow speeds, they found decreasing pressure loss coefficients with increasing speed, reaching a constant value for $u \approx 8 \mathrm{~m} \mathrm{~s}^{-1}$. This decrease is primarily due to the deformation towards a more streamlined shape of the branch and leaf elements and secondly due to viscous effects (Stokes flow) for very low flow speeds. In summary, their measurements resulted in pressure loss coefficients $\lambda_{\mathrm{fs}}$ ranging from 0.4 to $13.4 \mathrm{~m}^{-1}$ with the vast majority between 
$1.0 \mathrm{~m}^{-1}<\lambda_{\mathrm{fs}}<3.0 \mathrm{~m}^{-1}$, which is in agreement with the full-scale pressure loss coefficients of the tree models.

The lattice-cage construction also enabled us to realize different tree-stand densities $\rho_{\mathrm{ts}}$ by filling every $n$th cell of the lattice cage with wadding material. The tree-stand density is defined as a dimensionless number, $\rho_{\mathrm{ts}}=[0,1]$, indicating the relative fraction of the treeavenue occupied by trees. In the present study, two tree-stand densities $\rho_{\mathrm{ts}}$ were realized: (i) $\rho_{\mathrm{ts}}=1.0$, where each cell of the lattice cage was filled with wadding material (herein referred to as high stand density), and (ii) $\rho_{\mathrm{ts}}=0.5$, where every second cell was filled (herein referred to as low stand density).

The two dimensionless parameters, the crown porosity $P_{\mathrm{Vol}}$ and the tree-stand density $\rho_{\mathrm{ts}}$, both characterize the tree-avenue configuration. The fraction of the street canyon occupied by the volume of vegetation increases with decreasing crown porosity and increasing treestand density. This motivates the definition of a new parameter based on $P_{\mathrm{Vol}}$ and $\rho_{\mathrm{ts}}$, which is an integral measure for the total volume of vegetation of the avenue of trees. A suitable definition is given by

$$
a_{\mathrm{p}}=\rho_{\mathrm{ts}}\left(100-P_{\mathrm{Vol}}\right)
$$

where $P_{\mathrm{Vol}}$ is in (\%). The parameter $a_{\mathrm{p}}$ is henceforth named the avenue parameter. For the avenues of trees investigated herein $a_{\mathrm{p}}$ assumes values from 1.25 to 4.0 , and for a tree-free street canyon $a_{\mathrm{p}}=0$. It must be noted that the avenue parameter does not specify a unique avenue of trees, since avenues of trees differing in crown porosity and tree-stand density may have the same $a_{\mathrm{p}}$ value.

\section{Traffic Pollutant Dispersion and Concentrations}

This section summarizes the key findings from the wind-tunnel studies obtained with the street-canyon representations and tree models described in the forgoing section. General flow and dispersion mechanisms are discussed and the normalized wall-average, as well as the wall-maximum concentrations are presented. In the following subsections the dependency of pollutant dispersion and concentration on the wind direction and the street-canyon aspect ratio (Sect. 3.1), the influence of the crown porosity (Sect. 3.2) and the tree-stand density (Sect. 3.3) are discussed. An overview of all the investigated street-canyon/tree-avenue configurations is given in Table 1 or can be found in the internet database CODASC - Concentration Data of Street Canyons (CODASC 2008).

Table 1 Overview of investigated street-canyon/tree-avenue configurations

\begin{tabular}{|c|c|c|c|c|c|c|c|}
\hline & \multirow{2}{*}{$\begin{array}{l}\alpha \\
W / H\end{array}$} & \multicolumn{2}{|l|}{0} & \multicolumn{2}{|c|}{45} & \multicolumn{2}{|c|}{90} \\
\hline & & 1 & 2 & 1 & 2 & 1 & 2 \\
\hline & Tree-free & $\mathrm{x}$ & $\mathrm{x}$ & $\mathrm{x}$ & $\mathrm{x}$ & $\mathrm{x}$ & $\mathrm{x}$ \\
\hline \multirow[t]{2}{*}{$\rho_{\mathrm{ts}}=0.5$} & $\lambda=80\left(P_{\mathrm{Vol}}=97.5 \%\right)$ & $\mathrm{x}$ & - & $\mathrm{x}$ & $\mathrm{x}$ & $\mathrm{x}$ & $\mathrm{x}$ \\
\hline & $\lambda=200\left(P_{\mathrm{Vol}}=96.0 \%\right)$ & $\mathrm{x}$ & $\mathrm{x}$ & $\mathrm{x}$ & $\mathrm{x}$ & $\mathrm{x}$ & $\mathrm{x}$ \\
\hline \multirow[t]{2}{*}{$\rho_{\mathrm{ts}}=1.0$} & $\lambda=80\left(P_{\mathrm{Vol}}=97.5 \%\right)$ & $\mathrm{x}$ & - & $\mathrm{x}$ & $\mathrm{x}$ & $\mathrm{x}$ & $\mathrm{x}$ \\
\hline & $\lambda=200\left(P_{\mathrm{Vol}}=96.0 \%\right)$ & $\mathrm{x}$ & $\mathrm{x}$ & $\mathrm{x}$ & $\mathrm{x}$ & $\mathrm{x}$ & $\mathrm{x}$ \\
\hline
\end{tabular}




\subsection{Dependency on Wind Direction and Street-Canyon Aspect Ratio}

The flow topology within a street canyon and consequently the pollutant dispersion are primarily governed by the angle of the approaching flow. Depending on the angle, the street canyon is to a greater or lesser extent obstructed by the flanking buildings or aligned to the approaching wind (Fig. 1). In this study, three wind directions have been investigated, namely $\alpha=90^{\circ}, 45^{\circ}$ and $0^{\circ}$ corresponding to a perpendicular, oblique and parallel wind direction with respect to the street axis. Furthermore, the flow topology is also influenced by the street-canyon aspect ratio. Depending on the aspect ratio, the street-level area is to a greater or lesser extent decoupled from the above roof-level area and different vortex regimes develop (e.g. Baik and Kim 1999). In this study, two aspect ratios, $W / H=1$ and 2 , have been investigated for a canyon with street-length to building-height ratio $L / H=10$.

\subsubsection{Perpendicular Wind Direction $\left(\alpha=90^{\circ}\right)$}

The case of a perpendicular wind direction has been most frequently addressed in street-canyon-dispersion studies so far, since the flanking buildings obstruct the street canyon strongly from the approaching flow and the traffic pollutant concentrations are expected to be maximal. A flow regime develops that consists of a canyon vortex in the inner area and of corner eddies in the sideward street-canyon areas (Hunter et al. 1991). As a representative example for the traffic pollutant concentration distribution along the canyon walls for a perpendicular approaching wind, contour plots of the normalized wall concentrations in a tree-free street canyon and with two rows of avenues of trees are shown in Fig. 4. Since the flow and geometry conditions are symmetric with respect to the $x-z$ plane, only half of each wall is shown.

Maximum traffic pollutant concentrations occur in the inner area at the pedestrian level along the leeward wall A. The concentrations at the windward wall B are between 2 and 4 times lower than at the leeward wall $\mathrm{A}$. The concentrations decrease at the sideward canyon areas due to the enhanced ventilation by laterally entrained air. Here, a superposition of the canyon vortex and the corner eddy results in a helical flow structure transporting air towards the canyon inner area. The street-canyon aspect ratio $(W / H=1$ or 2$)$ affects the concentration level but does not substantially alter the flow and dispersion characteristics. The concentration distribution at the canyon walls is similar, however, with lower normalized pollutant
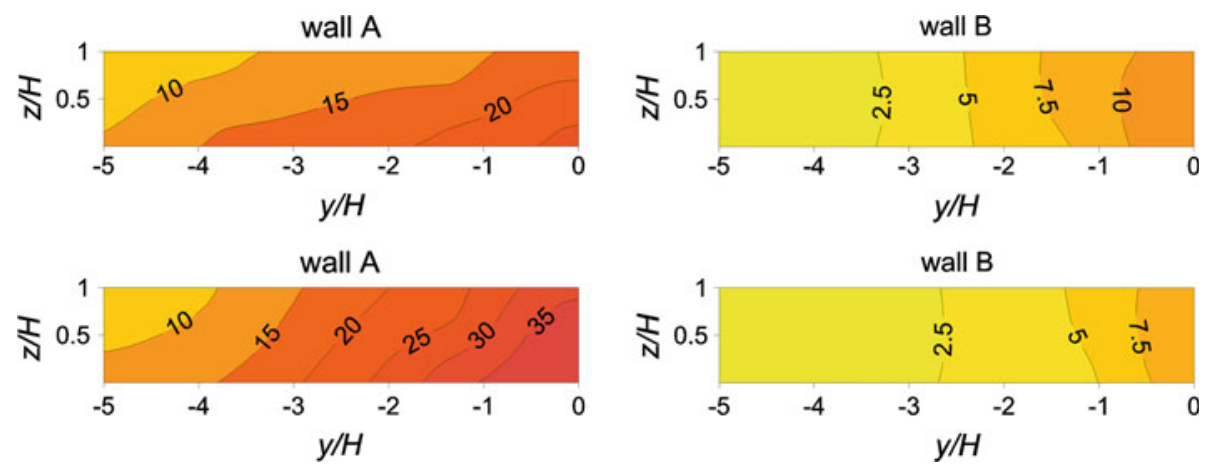

Fig. 4 Normalized pollutant concentrations $c^{+}$in a street canyon with $W / H=2$ subjected to a perpendicular wind direction $\left(\alpha=90^{\circ}\right)$. Top tree-free, bottom with two rows of tree-avenues of high stand density $\left(\rho_{\mathrm{ts}}=1.0\right)$ and low crown porosity $\left(P_{\mathrm{Vol}}=96 \%\right)$ 
concentrations in the broad street canyon. In this case, the lateral flow component induced by the corner eddies penetrates deeper towards the street-canyon inner area. This is reflected by the steadily increasing pollutant concentrations at each height level $(z / H=$ const) for $-5<y / H<0$, whereas in the narrow canyon a constant concentration level is established in the inner area at $z / H=$ constant. It should be noted that the normalized concentrations, i.e. the measured concentrations normalized by the emission-source strength according to Eq. 1, are lower in the broad street canyon. This does not necessarily imply lower traffic pollutant concentration levels in real broad street canyons, since in general the traffic volumes and consequently the emission-source strengths are larger. Avenues of trees affect the flow speeds and concentration levels but do not suppress totally the characteristic flow regime in the street canyons. In summary, they lead to higher traffic pollutant concentrations and lower flow speeds inside the street canyon with increases in traffic pollutant concentrations at the wall A and rather slight decreases at the wall B. Due to a blocking of the corner eddies by the tree crowns, stronger relative increases in concentrations occur in the sideward canyon areas (Gromke et al. 2008; Balczó et al. 2009; Buccolieri et al. 2009; Gromke and Ruck 2009b).

\subsubsection{Oblique Wind Direction $\left(\alpha=45^{\circ}\right)$}

For oblique wind directions (e.g. $\alpha=45^{\circ}$ ), a corkscrew flow regime develops due to the superposition of a street-axis parallel and perpendicular flow component (Soulhac et al. 2008; Buccolieri et al. 2011). Figure 5 shows a typical concentration distribution at the canyon walls for an oblique wind direction with $\alpha=45^{\circ}$.
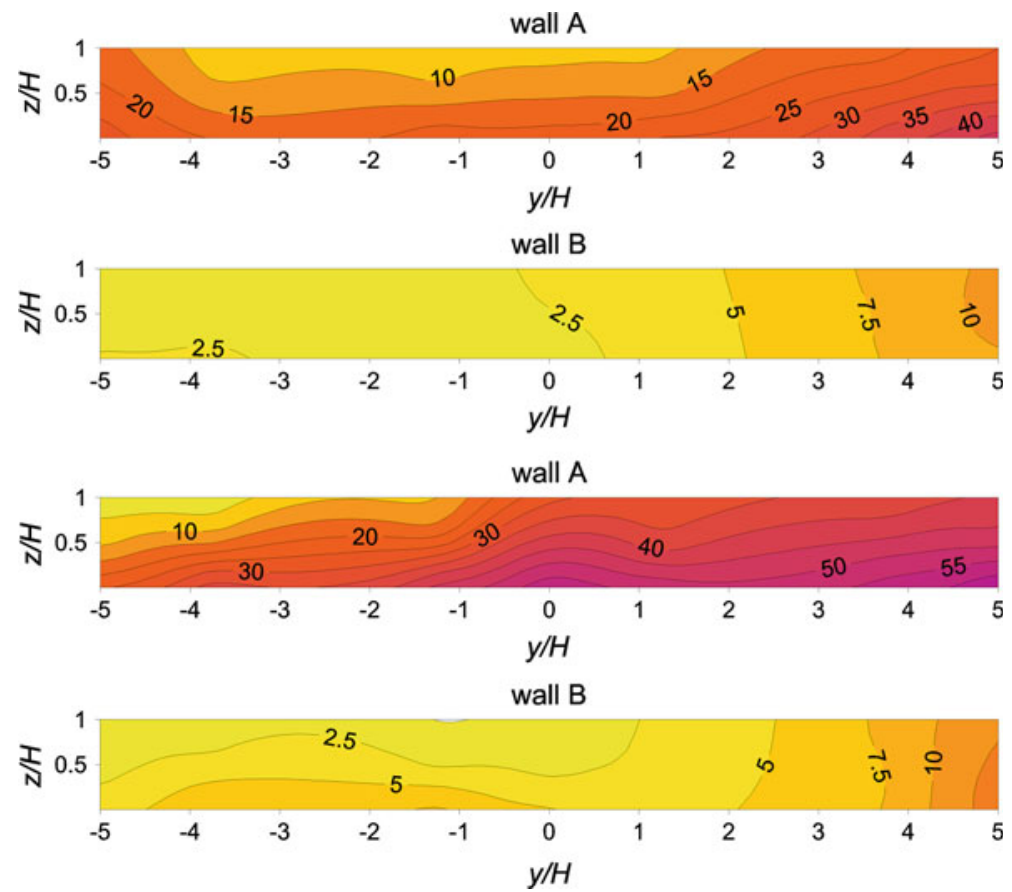

Fig. 5 Normalized pollutant concentrations $c^{+}$in a street canyon with $W / H=1$ subjected to an oblique wind direction $\left(\alpha=45^{\circ}\right)$. Top tree-free, bottom with on row of tree-avenue of low stand density $\left(\rho_{\mathrm{ts}}=0.5\right)$ and high crown porosity $\left(P_{\mathrm{Vol}}=97.5 \%\right)$ 

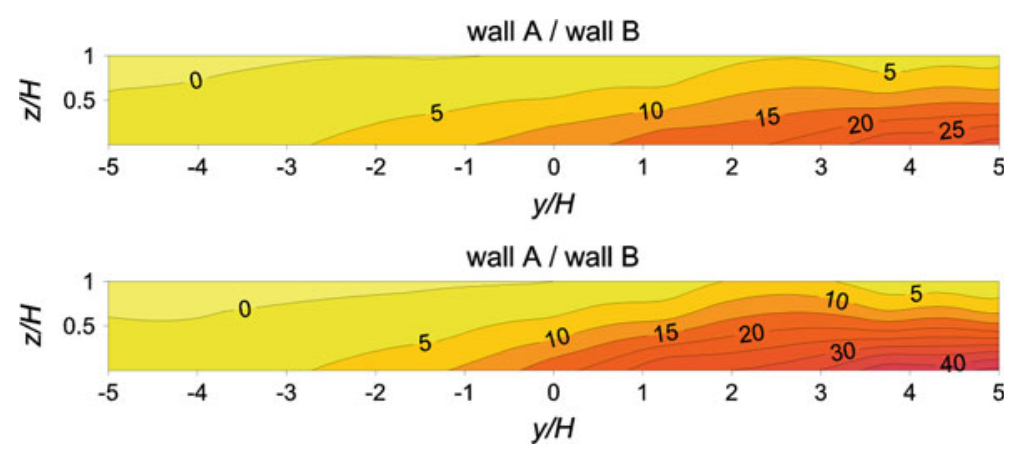

Fig. 6 Normalized pollutant concentrations $c^{+}$in a street canyon with $W / H=1$ subjected to a parallel wind direction $(\alpha=0)$. Top tree-free, bottom: with one row of tree-avenue of high tree-stand density $\left(\rho_{\mathrm{ts}}=1.0\right)$ and low crown porosity $\left(P_{\mathrm{Vol}}=96 \%\right)$

In this flow regime, traffic pollutants are transported and accumulated along the street axis. As a result, the concentrations increase towards the street-canyon end with a higher pollutant level at the leeward oriented wall A (Kastner-Klein and Plate 1999; Gromke 2008). The maximum concentrations occur at the pedestrian level at the canyon end area along the wall A. The experiments reveal corkscrew flow regimes for both aspect ratios investigated, but with markedly lower normalized pollutant concentrations for the broader street canyon. A corkscrew flow regime and its characteristic pollutant concentration distribution prevail also in the presence of avenues of trees. However, the avenues of trees result in distinct changes in concentration levels that exceed the tree-induced increases for a perpendicular wind direction (Gromke 2008; Buccolieri et al. 2011).

\subsubsection{Parallel Wind Direction $\left(\alpha=0^{\circ}\right)$}

Finally, for a parallel wind direction, the flanking buildings are aligned with the approaching wind direction. In Fig. 6, a representative example for a traffic pollutant concentration distribution for a parallel wind direction is shown. Since the flow and geometry conditions are symmetric with respect to the $y-z$ plane, only one wall is presented.

Independent of the aspect ratio, the flow regime within the canyon is dominated by a street-axis parallel flow. The parallel flow accumulates traffic pollutants and leads to a steady increase along the street axis with maximum concentration at the pedestrian level (Kastner-Klein and Plate 1999; Gromke 2008). Compared to a perpendicular or oblique wind direction, lower pollutant concentrations are found for the street-canyon geometry investigated here $(L / H=10)$. Larger aspect ratios result in strongly decreased normalized concentrations. The experimental results indicate that the parallel flow regime remains basically unaffected by avenues of trees (Gromke 2008). However, whereas for the narrow street canyon $(W / H=1)$ noticeable tree-induced increases in normalized traffic pollutant concentrations at the canyon walls are found, almost no changes are evident for the broad canyon $(W / H=2)$.

\subsection{Influence of the Tree-Crown Porosity}

Figure 7 shows the normalized wall-average $c_{\mathrm{ave}}^{+}$and wall-maximum $c_{\max }^{+}$traffic pollutant concentrations for the canyon walls $\mathrm{A}$ and $\mathrm{B}$ in dependency on the crown porosity $P_{\mathrm{Vol}}$ 

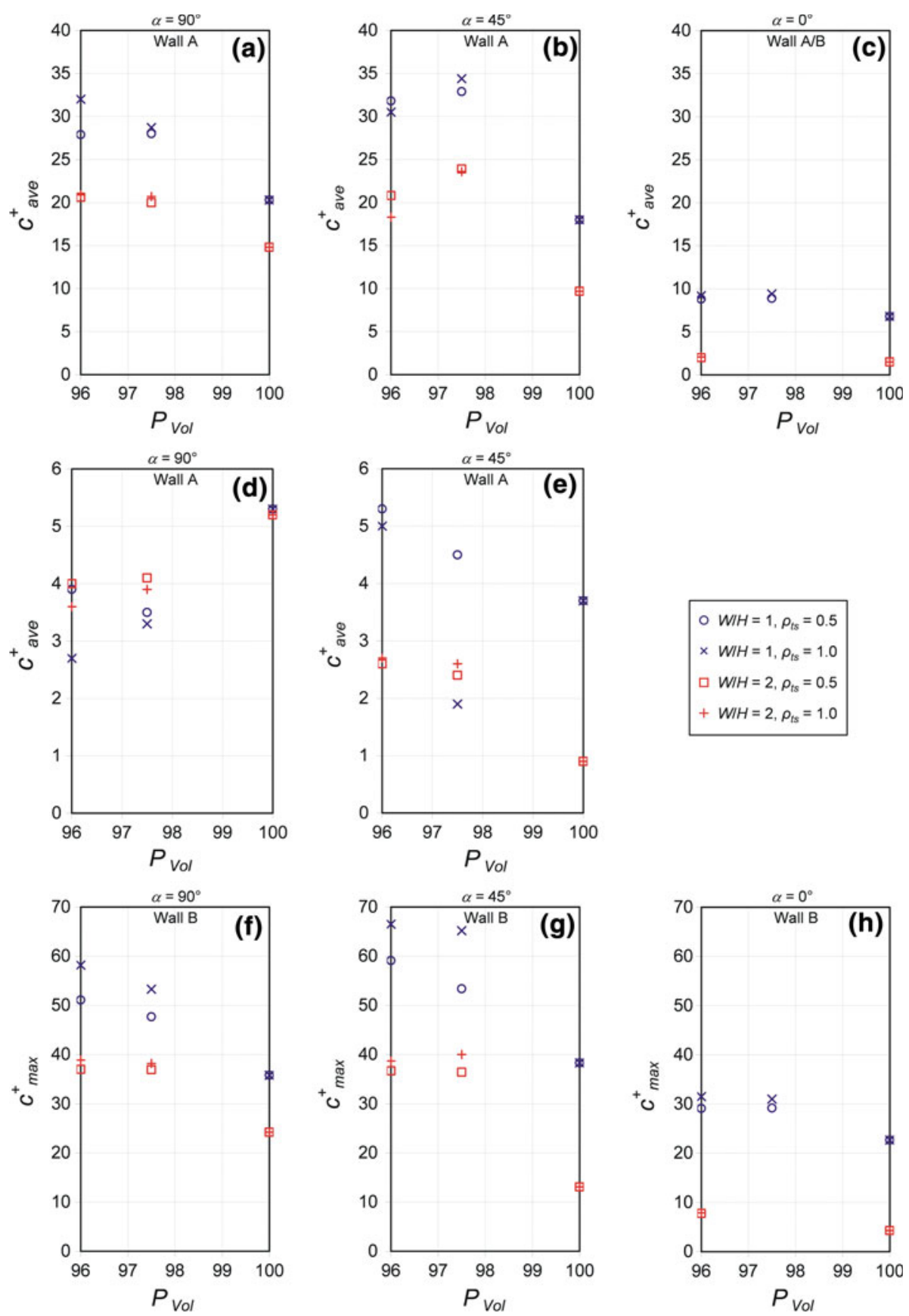

Fig. 7 Normalized wall-average concentrations $c_{\text {ave }}^{+}$in dependency on crown porosity $P_{\text {Vol }}$ at wall A (a-c) and wall $\mathrm{B}(\mathbf{c}-\mathbf{e})$, and normalized wall-maximum concentrations $c_{\max }^{+}$at wall $\mathrm{A}(\mathbf{f}-\mathbf{h})$ for a perpendicular $\left(\alpha=90^{\circ}\right)$, oblique $\left(\alpha=45^{\circ}\right)$ and parallel $(\alpha=0)$ approaching flow 
$\left(P_{\mathrm{Vol}}=100 \%\right.$ denotes the tree-free street canyon). The data are clustered in groups of constant aspect ratio $W / H$ and stand density $\rho_{\mathrm{ts}}$.

\subsubsection{Perpendicular Wind Direction $\left(\alpha=90^{\circ}\right)$}

For a perpendicular wind direction increasing normalized wall-average concentrations $c_{\mathrm{ave}}^{+}$ with decreasing crown porosity $P_{\mathrm{Vol}}$ are found at the wall A (Fig. 7a). In contrast, at wall B, in general decreasing wall-average concentrations $c_{\text {ave }}^{+}$with decreasing crown porosity $P_{\text {Vol }}$ are found (Fig. $7 \mathrm{~d}$ ). The maximum relative changes in wall-average concentrations at the wall A referred to the tree-free canyons are 58 and $41 \%$ in the case of the narrow $(W / H=1)$ and broad $(W / H=2)$ street canyon, respectively. The wind-tunnel experiments of Gromke and Ruck (2007, 2009b), as well as the numerical simulations of Gromke et al. (2008) and Buccolieri et al. (2009) indicate a flow field dominated by a canyon vortex and corner eddies in the presence of avenues of trees. However, the trees induce lower flow speeds inside the street canyon and modify the air exchange at the roof level. The concentration increases at wall A can be attributed to the lower flow speeds and reduced air-mass flows, whereas the decreases at wall B originate in the modified air exchange. In a tree-free street canyon, the canyon vortex entrains air at the roof level and directs the flow downward in front of the windward wall $\mathrm{B}$. When passing the street level, the canyon vortex accumulates traffic exhausts and leads to high concentrations at the leeward wall A where the air flow is directed upward. At the roof level, the air exchange with the above roof wind dilutes the pollutant concentration in the canyon vortex before the air is re-entrained in the front of wall B. In a street canyon with avenues of trees, the upward flow is in a way channelled between the avenues of trees and wall $\mathrm{A}$, and penetrates deeper into the above-roof flow with resulting enhanced air exchange. Consequently, the re-entrained air in front of wall B is less polluted. Furthermore, the avenues of trees hinder a direct transport of traffic pollutants towards wall B.

The diagrams (Fig. 7a, d) reveal, in general, degressive changes in wall-average concentrations $c_{\text {ave }}^{+}$with decreasing crown porosity $P_{\mathrm{Vol}}$ for both walls. Except for the narrow street canyon with high stand density $\left(W / H=1, \rho_{\mathrm{ts}}=1\right)$, no distinct differences in the concentration levels between the high and low crown porosity are found. Concentration measurements with non-porous tree models $\left(P_{\mathrm{Vol}}=0 \%\right)$ by Gromke and Ruck $(2009 \mathrm{~b})$ show no notable differences compared to tree models with low crown porosity $\left(P_{\mathrm{Vol}}=96 \%\right)$. This implies that the low porosity crown behaves virtually as a non-porous object and further suggests that remarkable changes in pollutant dispersion occur only at higher crown porosities, i.e. $P_{\mathrm{Vol}}>97.5 \%$. The latter statement is substantiated by flow studies past fences or vegetation shelterbelts (e.g. Gandemer 1981; Grant and Nickling 1998; Frank and Ruck 2005) where a strong sensitivity of the flow resistance is only observed in the high porosity range.

The normalized wall-maximum concentrations $c_{\max }^{+}$at the wall A (Fig. 7f) increase steadily with decreasing crown porosity $P_{\mathrm{Vol}}$ for the narrow street canyon. However, for the broad canyon, the normalized wall-maximum concentrations appear to have reached their maximum level already with the high crown porosity $P_{\mathrm{Vol}}=97.5 \%$. The largest relative increases due to avenues of trees are of the same magnitude for both street-canyon aspect ratios, with $63 \%$ for the narrow and $61 \%$ for the broad street canyon.

\subsubsection{Oblique Wind Direction $\left(\alpha=45^{\circ}\right)$}

For an oblique wind direction no monotonic relation between the normalized wall-average concentration $c_{\text {ave }}^{+}$and the crown porosity can be identified at wall A (Fig. 7b). With reference 
to the tree-free street canyon, the strongest increases in concentrations are found for the high porosity instead of for the low porosity tree-avenues, with relative changes at wall A of 91 and $146 \%$ for the narrow and broad street canyon, respectively. This is a very interesting finding since the tree-avenues are denser in the case of the lower crown porosity and one is lead to expect increasing traffic pollutant concentrations. A possible explanation for this is a more effective street axis parallel channelling of the flow where the flow enters the street canyon $(y / H=-5)$ in the presence of tree-avenues with low crown porosity and that inhibits a direct transport of traffic pollutants towards wall A. This hypothesis is substantiated by the concentration contour plot for the tree-avenue with low crown porosity shown in Fig. 8. In comparison to the corresponding tree-avenue with high crown porosity (Fig. 5), lower pollutant concentrations at the wall A for $y / H<-1$ are observed. However, the hypothesis cannot be finally proven on the basis of the available data. Biases resulting from measurement errors or an inaccurate model set-up are excluded because of the clear trend that can be seen in all the configurations.

The wall-average concentrations at the wall B show again a monotonic behaviour (Fig. 7e). With the exception of the data point for $W / H=1$ and $\rho_{\mathrm{ts}}=1$, which is not considered to be reliable, increasing concentration levels with decreasing porosity are present. This indicates the dominance of the street-axis parallel flow component, which transports and accumulates traffic emissions, over the perpendicular flow component since otherwise decreasing concentrations with decreasing crown porosity, as for a perpendicular wind direction, would prevail. However, even with the avenues of trees, the concentration levels at the wall B remain still comparatively low.

The normalized wall-maximum concentrations $c_{\max }^{+}$(Fig. $7 \mathrm{~g}$ ) do not reflect the non-monotonic relation with crown porosity which is found for the wall-average concentrations at wall A. Instead, continuously increasing concentrations with decreasing porosity are observed with relative changes of 74 and $205 \%$ referred to the narrow and broad tree-free canyon, respectively. As can be seen from Fig. $7 \mathrm{f}-\mathrm{h}$, the largest wall-maximum traffic pollutant concentrations in the narrow street canyon occur for an oblique wind direction rather than for a perpendicular wind direction. This is a new and very interesting finding. It disproves the common assumption in street-canyon dispersion studies that attributes the most critical pollutant concentration levels to a perpendicular wind direction. Clearly, oblique wind directions only become more critical in relative long street canyons, i.e. when the aspect ratio $L / H$ exceeds a certain threshold. An analysis of the wind-tunnel data (CODASC 2008) shows that this threshold depends on the aspect ratio and the characteristics of the avenues of trees. Table 2 contains the $L / H$ thresholds for the investigated street-canyon/tree-avenue configurations. The threshold estimation is based on the $y / H$ position in the street canyon where the near-ground pollutant concentrations at wall $\mathrm{A}$ for an oblique wind direction $\left(\alpha=45^{\circ}\right)$ exceed the wall-maximum

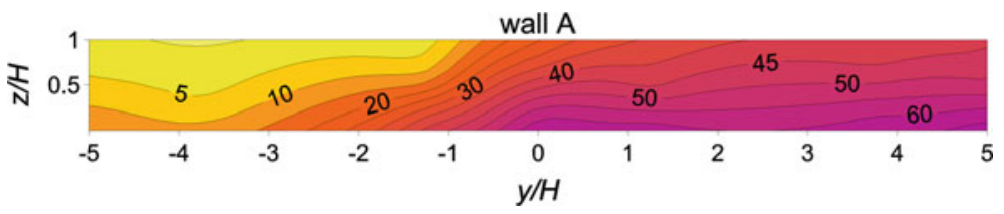

Fig. 8 Normalized pollutant concentrations $c^{+}$in a street canyon with $W / H=1$ subjected to an oblique wind direction $\left(\alpha=45^{\circ}\right)$ with one row of tree-avenue of low stand density $\left(\rho_{\mathrm{ts}}=0.5\right)$ and low crown porosity $\left(P_{\mathrm{Vol}}=96 \%\right)$. Compare with Fig. 5 
Table $2 L / H$ thresholds when the wall-maximum concentration $c_{\max }^{+}$for an oblique wind direction $\left(\alpha=45^{\circ}\right)$ exceeds the wall-maximum concentration $c_{\max }^{+}$for a perpendicular wind direction $\left(\alpha=90^{\circ}\right)$

\begin{tabular}{lllll}
\hline$W / H$ & $\rho_{\mathrm{ts}}$ & \multicolumn{2}{l}{$P_{\mathrm{Vol}}(\%)$} & $97.5 \%$ \\
\cline { 3 - 5 } & & $96 \%$ & 9.5 & $100 \%$ \\
\hline 1 & 0.5 & 7.5 & 7.5 & 9.5 \\
& 1.0 & 5 & $>10$ & $>10$ \\
& 0.5 & 9 & 9 & \\
\hline
\end{tabular}

$P_{\mathrm{Vol}}=100 \%$ denotes the tree-free street canyon

concentration $c_{\max }^{+}$of the corresponding street-canyon/tree-avenue configuration for a perpendicular flow.

It can be seen that the $L / H$ threshold is always smaller for the narrow street canyon and for the high stand density, and generally decreases with decreasing crown porosity. The common assumption that attributes the most critical pollutant concentration levels to a perpendicular wind direction is especially not valid in narrow street canyons with dense avenues of trees (i.e. high stand density and low crown porosity). Here, even in rather short street canyons, an oblique wind direction results in higher wall-maximum concentrations. It has to be noted that the analysis of the wall-maximum concentration and of the $L / H$ threshold refers to an oblique wind direction with $\alpha=45^{\circ}$. Higher wall-maximum traffic pollutant concentrations may occur for oblique wind directions approaching for angles other than $\alpha=45^{\circ}$ and the $L / H$ thresholds may be smaller.

\subsubsection{Parallel Wind Direction $\left(\alpha=0^{\circ}\right)$}

Figure $7 \mathrm{c}$ and $\mathrm{h}$ shows increased normalized wall-average and wall-maximum concentrations in street canyons with tree-avenues of lower crown porosity for a parallel wind direction. The largest relative increases in the narrow and broad street canyon amount to 38 and $40 \%$ for the wall-average and to 39 and $84 \%$ for the wall-maximum concentration. However, in comparison to the other wind directions, the concentration levels are considerably smaller, in particular in the broad street canyon. For the narrow street canyon, no differences between the low and high crown porosity can be found, suggesting that there is no further increase in concentration levels with denser tree crowns. Overall, the results indicate that a parallel wind direction is practically not relevant in causing high normalized wall-average and wall-maximum concentrations.

\subsection{Influence of Tree-Stand Density}

Figure 9 shows the normalized wall-average $c_{\text {ave }}^{+}$and wall-maximum $c_{\max }^{+}$traffic pollutant concentrations for the canyon walls $\mathrm{A}$ and $\mathrm{B}$ as a function of the stand density $\rho_{\mathrm{ts}}\left(\rho_{\mathrm{ts}}=0\right.$ denotes the tree-free street canyon). The data are clustered in groups of constant aspect ratio $W / H$ and crown porosity $P_{\mathrm{Vol}}$.

\subsubsection{Perpendicular Wind Direction $\left(\alpha=90^{\circ}\right)$}

For a perpendicular wind direction, the normalized wall-average concentrations $c_{\text {ave }}^{+}$increase at wall A (Fig. 9a) and decrease at wall B (Fig. 9d) with increasing stand density $\rho_{\text {ts. }}$. 

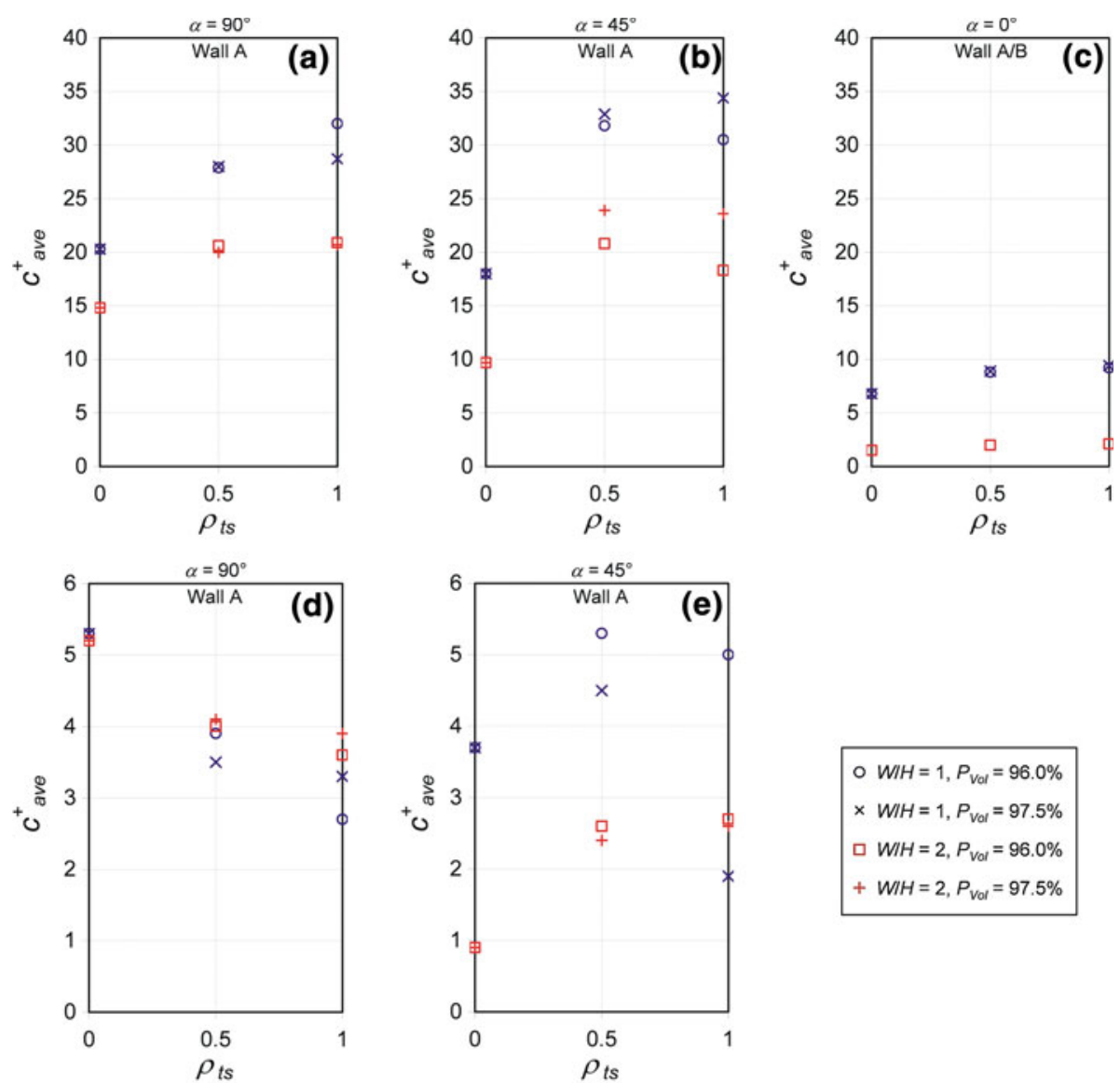

$\circ W H=1, P_{\text {Val }}=96.0 \%$
$\times W H=1, P_{\text {Vol }}=97.5 \%$
$\square W H=2, P_{\text {Vol }}=96.0 \%$
$+W H=2, P_{\text {Vol }}=97.5 \%$
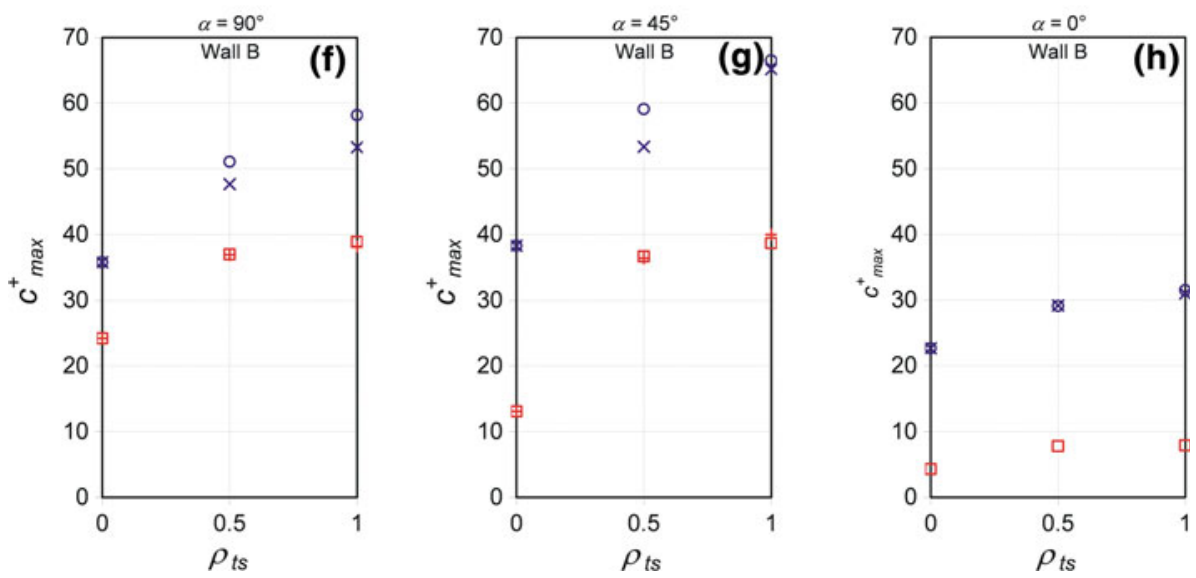

Fig. 9 Normalized wall-average concentrations $c_{\text {ave }}^{+}$in dependency on stand density $\rho_{\text {ts }}$ at wall A (a-c) and wall $\mathrm{B}(\mathbf{c}-\mathbf{e})$, and normalized wall-maximum concentrations $c_{\max }^{+}$at wall $\mathrm{A}(\mathbf{f}-\mathbf{h})$ for a perpendicular $\left(\alpha=90^{\circ}\right)$, oblique $\left(\alpha=45^{\circ}\right)$ and parallel $\left(\alpha=0^{\circ}\right)$ approaching flow 
Degressive changes in the wall-average concentrations $c_{\text {ave }}^{+}$with increasing stand density $\rho_{\mathrm{ts}}$ at the wall A can be identified. With the exception of the narrow street canyon with low crown porosity, no distinct differences in the concentration level between the high and low stand density can be found. The wall-maximum concentrations $c_{\max }^{+}$at wall A (Fig. 9f) increase steadily with increasing stand density $\rho_{\mathrm{ts}}$. These relationships resemble those identified between the wall-average concentration and the crown porosity in Sect. 3.2.1 for which with lower crown porosity, i.e. with increasingly denser tree-avenues, the same characteristics are observed.

It has to be critically noted that for the low stand density $\rho_{\mathrm{ts}}=0.5$, although every second cage cell is not filled with wadding material, the realization of the tree models by means of the continuous lattice-cage construction has some influence on the measurement results (Fig. 2). Experiments with an empty lattice cage in the street canyon by Gromke and Ruck (2009b) resulted in altered traffic pollutant concentrations in the order of $20 \%$ compared to the tree-free situation (increase at the wall A and decrease at the wall B). However, regardless of this bias, the tendency of the impacts of spaced-out avenues of trees on traffic pollutant dispersion and concentrations in urban street canyons is clearly visible.

\subsubsection{Oblique Wind Direction $\left(\alpha=45^{\circ}\right)$}

For an oblique wind direction, in general, the relation between the normalized wall-average concentration $c_{\text {ave }}^{+}$and stand density $\rho_{\mathrm{ts}}$ at wall A shows no monotonic characteristic (Fig. 9b). In the broad street canyon, higher normalized concentration levels are found for the low stand density instead of for the high stand density avenues of trees, whereas in the narrow canyon, this trend is only visible for the low crown porosity avenues of trees. Likewise with the crown porosity (Sect. 3.2.2), denser avenues of trees do not lead to higher wall-average traffic pollutant concentrations. This phenomenon is also attributed to the more effective street-axis parallel channelling of the flow in the presence of denser tree-avenues as described earlier.

The normalized wall-average concentrations at the wall B show different characteristics depending on the street-canyon aspect ratio (Fig. 9e). In the narrow street canyon, a nonmonotonic relation between the wall-average concentration and the stand density can be seen, whereas in the broad canyon a slight concentration increase for the high stand density relative to the low stand density can be found.

The wall-maximum concentrations $c_{\max }^{+}$show always a continuous increase with increasing stand density (Fig. 9g), with the largest wall-maximum concentrations occurring in the presence of the high stand density avenues of trees. A comparison between the two aspect ratios reveals a marked sensitivity of the normalized wall-maximum concentrations to the stand density for $\rho_{\mathrm{ts}}<0.5$ in the broad street canyon. This indicates that spacing-out trees is a very effective means of reducing the wall-maximum concentrations in broad street canyons.

\subsubsection{Parallel Wind Direction $\left(\alpha=0^{\circ}\right)$}

In street canyons with avenues of trees increased normalized wall-average and wall-maximum concentrations for a parallel wind direction are found (Fig. 9c, h). However, the differences between the low and high stand densities are rather small. In comparison to the other wind directions, the normalized concentration levels are considerably smaller, in particular in the broad street canyon. These observations substantiate the hypothesis stated earlier for the crown porosity (Sect. 3.2.3) that a parallel wind direction is not practically relevant and does not result in high normalized wall-average or wall-maximum concentrations. 


\section{Dimensional Analysis and Wall-Maximum Concentrations}

The final goal of the dimensional analysis is to provide an estimate of the normalized wallmaximum traffic pollutant concentration for the street-canyon/tree-avenue configurations discussed in Sect. 3 and published in the internet database CODASC (CODASC 2008). The wall-maximum concentration is of particular interest in urban air quality and town planning. As the configurations comprise different street-canyon geometries, tree-avenue arrangements and approaching wind directions, the resulting estimate is deemed representative of a wide range of urban street canyons. The maximum pollutant concentration refers here to the long term rather than to short term maximum concentrations since mean values were measured in the wind-tunnel experiments.

\subsection{Dimensional Analytical Considerations}

For the dimensional analysis, all parameters that possibly influence the physics and dynamics of the system under investigation have to be identified first. For the isolated generic streetcanyon/tree-avenue configurations investigated in this study, the following parameters are relevant for the traffic pollutant dispersion:

- Geometry parameters: building height $H(\mathrm{~m})$, street-canyon length $L(\mathrm{~m})$, street-canyon width $W(\mathrm{~m})$, roof geometry $\mathbf{r}_{\mathrm{g}}(\mathrm{m})$, see Fig. 1

- Tree-avenue parameters: stand density $\rho_{\mathrm{ts}}(-)$, crown porosity $P_{\mathrm{Vol}}(-)$, positions of trees $\mathbf{x}_{\mathrm{t}}(\mathrm{m})$, crown geometry $\mathbf{x}_{\mathrm{c}}(\mathrm{m})$

- Flow parameters: wind speed at roof level $u_{\mathrm{H}}\left(\mathrm{m} \mathrm{s}^{-1}\right)$, approaching wind direction $\alpha(-)$, kinematic viscosity $v\left(\mathrm{~m}^{2} \mathrm{~s}^{-1}\right)$

- Source parameters: emission strength $Q_{1}\left(\mathrm{~m}^{2} \mathrm{~s}^{-1}\right)$, line-source positions $\mathbf{x}_{\mathrm{ls}}(\mathrm{m})$

The mean flow and turbulence characteristics of the approaching flow are considered not essential, but when the approaching flow encounters an isolated street canyon, its structure is significantly perturbed. The flow and dispersion processes inside and in the proximity of isolated street canyons are governed by the canyon and building geometry, in particular by the roof geometry $\mathbf{r}_{\mathrm{g}}$ of the windward oriented building (Huang et al. 2009; Kastner-Klein and Plate 1999). Also the building widths $W_{\mathrm{A}}$ and $W_{\mathrm{B}}$ (Fig. 1) are considered not essential for the flow and pollutant dispersion inside the street canyons. For winds approaching other than parallel, the roof geometry of the windward building dominates the topology of the separated flow rather than the building width. A further assumption is that the two building blocks forming the street canyon have the same height $H$, which is widely the case for urban street canyons. It must be noted that the crown porosity $P_{\mathrm{Vol}}$ is used instead of the pressure loss coefficient $\lambda$, and involves only a formal but not a fundamental distinction since the crown porosity and the pressure loss coefficient of the tree models are related to each other.

Following these considerations, the traffic pollutant concentration $c(\mathbf{x})(-)$ at position $\mathbf{x}$ in an isolated street-canyon/tree-avenue configuration can be expressed by the general formulation

$$
c(\mathbf{x})=f\left(H, L, W, \mathbf{r}_{\mathrm{g}}, \rho_{\mathrm{ts}}, P_{\mathrm{Vol}}, \mathbf{x}_{\mathrm{t}}, \mathbf{x}_{\mathrm{c}}, u_{\mathrm{H}}, \alpha, v, Q_{1}, \mathbf{x}_{\mathrm{ls}}, \mathbf{x}\right) .
$$

Employing the Buckingham $\pi$-theorem (e.g. Stull 1988), the number $n$ of parameters can be reduced to $n-m$ dimensionless parameters, so called $\pi$-groups, with $m$ the number of base quantities. Equation 7 involves $m=2$ base quantities, the length $\{1\}$ and the time $\{\mathrm{t}\}$. Normalizing the parameters in Eq. 7 with $H$ as the length scale and $u_{\mathrm{H}}$ as combined length and time scales results in 


$$
c(\mathbf{x})=f\left(\frac{L}{H}, \frac{W}{H}, \frac{\mathbf{r}_{\mathrm{g}}}{H}, \rho_{\mathrm{ts}}, P_{\mathrm{Vol}}, \frac{\mathbf{x}_{\mathrm{t}}}{H}, \frac{\mathbf{x}_{\mathrm{c}}}{H}, \alpha, \operatorname{Re}_{\mathrm{H}}, \frac{Q_{\mathrm{l}}}{u_{\mathrm{H}} H}, \frac{\mathbf{x}_{\mathrm{ls}}}{H}, \frac{\mathbf{x}}{H}\right)
$$

where $R e_{\mathrm{H}}=u_{\mathrm{H}} H v^{-1}$.

According to these formal dimensional analytical considerations, the concentration $c(\mathbf{x})$ is Reynolds-number dependent. However, taking advantage of the fact of largely Reynoldsnumber independent flows around sharp edged geometries for $R e_{\mathrm{H}}>15,000$ (Meroney 2004), it is argued that the flow field and pollutant dispersion inside the street canyon $\left(R e_{\mathrm{H}}=37,000\right.$, see Sect. 2.1) are not functions of $R e_{\mathrm{H}}$. This in turn implies halved pollutant concentrations when the free-stream velocity $u_{\mathrm{H}}$ is doubled. Moreover, doubling the tracer-gas source strength $Q_{1}$ will clearly result in twice as high pollutant concentrations. Combining the two considerations, it is straightforward that the pollutant concentration linearly depends on $Q_{1} u_{\mathrm{H}}^{-1} H^{-1}$. Following these arguments, one arrives at

$$
c(\mathbf{x}) \frac{u_{\mathrm{H}} H}{Q_{1}}=c^{+}(\mathbf{x})=f\left(\frac{L}{H}, \frac{W}{H}, \frac{\mathbf{r}_{\mathrm{g}}}{H}, \rho_{\mathrm{ts}}, P_{\mathrm{Vol}}, \frac{\mathbf{x}_{\mathrm{t}}}{H}, \frac{\mathbf{x}_{\mathrm{c}}}{H}, \alpha, \frac{\mathbf{x}_{\mathrm{ls}}}{H}, \frac{\mathbf{x}}{H}\right) .
$$

Equation 9 is a general formulation for the normalized dimensionless concentration $c^{+}$, and the linear dependency in $Q_{1} u_{\mathrm{H}}^{-1} H^{-1}$ results in normalized concentrations $c^{+}$according to Eq. 1.

\subsection{Estimate of the Wall-Maximum Traffic Pollutant Concentrations}

Based on the dimensional analytical considerations and the wind-tunnel experiments, a relationship that allows to estimate the normalized maximum pollutant concentration $c_{\max }^{+}$at the canyon walls is derived. The entire wind-tunnel measurement dataset comprises 28 streetcanyon/tree-avenue configurations as discussed in Sect. 3 and is accessible in the internet database CODASC under www.codasc.de (CODASC 2008). An overview of the parameters of the configurations is given in Table 1. In addition, wind-tunnel experiments performed with empty lattice cages $\left(P_{\mathrm{Vol}}=99.0 \%\right.$ and $\left.\rho_{\mathrm{ts}}=1.0\right)$, see Gromke (2008) or Gromke and Ruck (2009b) are included for the derivation.

However, not all parameters contained in the dimensionless $\pi$-groups of Eq. 9 were varied systematically in the wind-tunnel experiments. Among them are the street-canyon length $L$, the roof geometry $\mathbf{r}_{\mathrm{g}}$, the positions of the trees $\mathbf{x}_{\mathrm{t}}$, the crown geometry $\mathbf{x}_{\mathrm{c}}$ and the line-source positions $\mathbf{x}_{\mathrm{ls}}$. The missing parameter variations prevent an explicit assessment of their influence on the normalized wall-maximum concentration $c_{\max }^{+}$. Instead, these parameters have to be considered as boundary conditions. Removing these parameters from Eq. 9 gives

$$
c^{+}(\mathbf{x})=f\left(\frac{W}{H}, \rho_{\mathrm{ts}}, P_{\mathrm{Vol}}, \alpha, \frac{\mathbf{x}}{H}\right)
$$

with the boundary conditions $L / H=10$, with flat-roof geometry $\mathbf{r}_{\mathrm{g}}$ and positions of trees $\mathbf{x}_{\mathrm{t}}$, crown geometry $\mathbf{x}_{\mathrm{c}}$ and line-source positions $\mathbf{x}_{\mathrm{ls}}$ depending on the $W / H$ ratio according to Fig. 1.

From an urban air quality and town planning point of view, the estimate does not necessarily need to provide any information on the location $\mathbf{x}$ of the wall-maximum concentration. More interesting is the value of $c_{\max }^{+}$rather than its location at the building walls. Consequently, Eq. 10 can be simplified to

$$
c_{\max }^{+}=f\left(\frac{W}{H}, \rho_{\mathrm{ts}}, P_{\mathrm{Vol}}, \alpha\right) .
$$




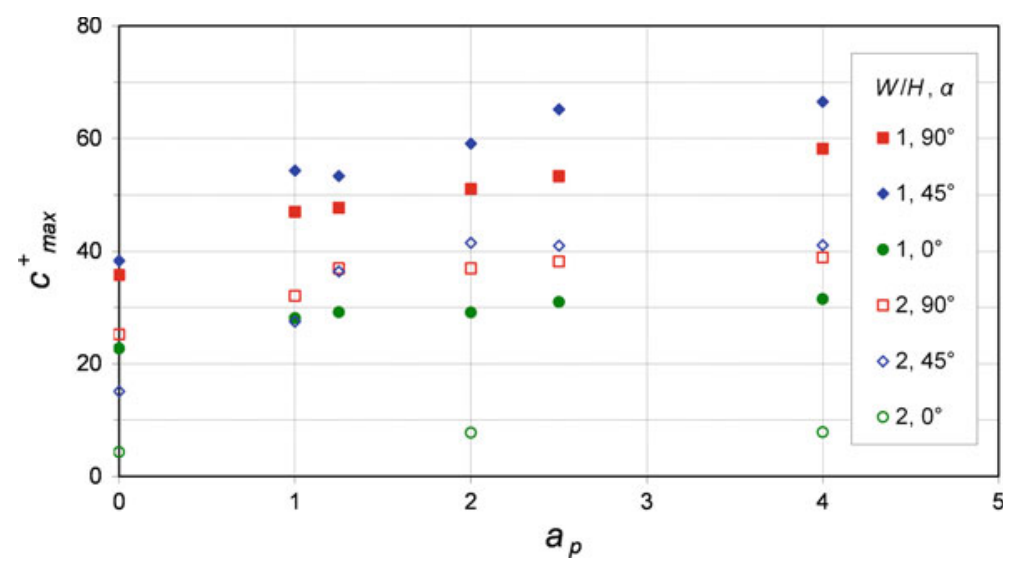

Fig. 10 Normalized wall-maximum pollutant concentrations versus the avenue parameter $a_{\mathrm{p}}$

Although Eq. 11 does not involve any explicit information on the location of the wall-maximum traffic pollutant concentration, it is empirically known from the wind-tunnel experiments that the wall-maximum concentrations are always in the street-level region at the leeward oriented wall, either in the canyon inner area when the wind is approaching perpendicular or at the canyon end for an oblique and parallel wind direction.

Equation 11 contains two dimensionless parameters characterizing the tree-avenue configuration, the stand density $\rho_{\mathrm{ts}}$ and the crown porosity $P_{\mathrm{Vol}}$. In Sect. 2 it is suggested by Eq. 6 to combine both parameters in the avenue parameter $a_{\mathrm{p}}=\rho_{\mathrm{ts}}\left(100-P_{\mathrm{Vol}}\right)$, which is an integral measure of the total volume of vegetation of the tree-avenues. The wind-tunnel measurements presented in Sect. 3 generally reveal increasing normalized wall-maximum concentration $c_{\max }^{+}$with decreasing crown porosity or increasing stand density, i.e. with increasing $a_{\mathrm{p}}$. This motivates to substitute the stand density $\rho_{\mathrm{ts}}$ and crown porosity $P_{\mathrm{Vol}}$ by the avenue parameter $a_{\mathrm{p}}$ in order to further simplify the derivation of an estimate of the normalized wall-maximum concentration. The general functional relationship for $c_{\max }^{+}$is now expressed as

$$
c_{\max }^{+}=f\left(\frac{W}{H}, a_{\mathrm{p}}, \alpha\right)
$$

where $a_{\mathrm{p}}=\rho_{\mathrm{ts}}\left(100-P_{\mathrm{Vol}}\right)$, according to Eq. 6 .

Figure 10 shows the normalized wall-maximum pollutant concentrations $c_{\max }^{+}$versus the avenue parameter $a_{\mathrm{p}}$ for the street-canyon/tree-avenue configurations presented in Sect. 3 and additionally for street canyons with empty lattice cages, i.e. $a_{\mathrm{p}}=1.0$ (Gromke 2008; Gromke and Ruck 2009b). For each group of $W / H=$ constant and $\alpha=$ constant, increases in wall-maximum pollutant concentration are evident for increasing avenue parameter, with progressively smaller incremental growth rates for larger values of $a_{\mathrm{p}}$. This suggests that the normalized wall-maximum concentrations $c_{\max }^{+}$asymptotically approach an upper limiting value with increasing avenue parameter.

The characteristic in normalized wall-maximum concentrations $c_{\max }^{+}$of progressively smaller incremental growth rates for larger $a_{\mathrm{p}}$ and the asymptotic behaviour is mathematically described by a function of the form

$$
c_{\max }^{+}=a_{1}-a_{2} \exp \left(-a_{3} a_{\mathrm{p}}\right)
$$


where $a_{1}, a_{2}$ and $a_{3}$ are functions of the aspect ratio $W / H$ and the approaching wind direction $\alpha$ still needing to be defined.

Equation 13 results in largest $c_{\max }^{+}$values for tree-avenues of high stand densities and low crown porosities (large $a_{\mathrm{p}}$ ) with the limiting value $c_{\max }^{+}=a_{1}$. For tree-avenues of low stand densities and high crown porosities (small $a_{\mathrm{p}}$ ), Eq. 13 results in minimal $c_{\max }^{+}$values with the limiting value $c_{\max }^{+}=a_{1}-a_{2}$ for the tree-free configurations. That is, $a_{2}$ gives the maximum difference in wall-maximum pollutant concentration for a fixed parameter pair of constant $W / H$ and $\alpha$. Finally, $a_{3}$ can be interpreted as a stretching factor, indicating how sensitive the wall-maximum pollutant concentration responses to variations in the avenue parameter $a_{\mathrm{p}}$. For the formulation of the functions $a_{i}=\mathrm{f}_{i}(W / H, \alpha)$ in Eq. 13 a general approach of mixed polynomials was chosen according to

$$
a_{i}=c_{i 1}+c_{i 2} \frac{W}{H}+c_{i 3} \alpha+c_{i 4} \alpha^{2}+c_{i 5} \frac{W}{H} \alpha+c_{i 6} \frac{W}{H} \alpha^{2}
$$

where $\alpha$ is in radians. Table 3 contains the coefficients $c_{i j}$ as determined by means of linear regression analysis employing the data of Fig. 10. The graphs of the functions $a_{i}$ after Eq. 14 are shown in Fig. 11 for the aspect ratios $W / H=1.0,1.5$ and 2.0.

The range of validity of Eq. 13 is defined by the boundary conditions of the wind-tunnel experiments and the underlying assumptions involved in its derivation. The estimate of the normalized wall-maximum traffic pollutant concentration $c_{\max }^{+}$given by Eq. 13 can be strictly applied for urban street canyons of aspect ratios $W / H=1$ and $W / H=2$ with $L / H=10$. In addition, the $W / H$ range may be interpolated and extended as long as the street-canyon flow field does not change significantly. The canyons include either a single avenue of trees

Table 3 Coefficients $c_{i j}$ for Eq. 14

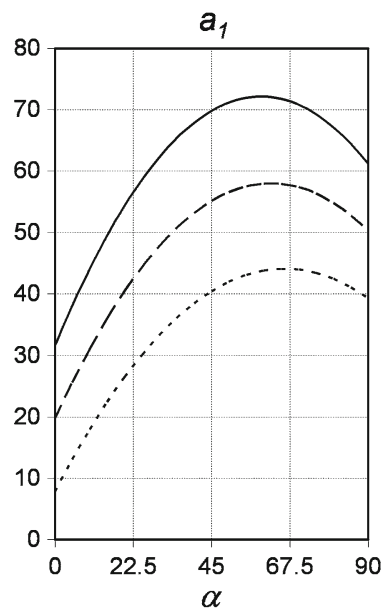

\begin{tabular}{rrrrrrr}
\hline $\mathrm{i}$ & \multicolumn{1}{c}{$c_{i 1}$} & \multicolumn{1}{c}{$c_{i 2}$} & \multicolumn{1}{c}{$c_{i 3}$} & \multicolumn{1}{c}{$c_{i 4}$} & \multicolumn{1}{c}{$c_{i 4}$} & \multicolumn{1}{c}{$c_{i 6}$} \\
\hline 1 & 55.3 & -23.8 & 94.2 & -48.7 & -15.5 & 10.7 \\
2 & 14.1 & -5.3 & 41.0 & -17.6 & 6.4 & -6.0 \\
3 & 0.0 & 0.9 & 0.3 & -0.2 & -0.8 & 0.4 \\
\hline
\end{tabular}
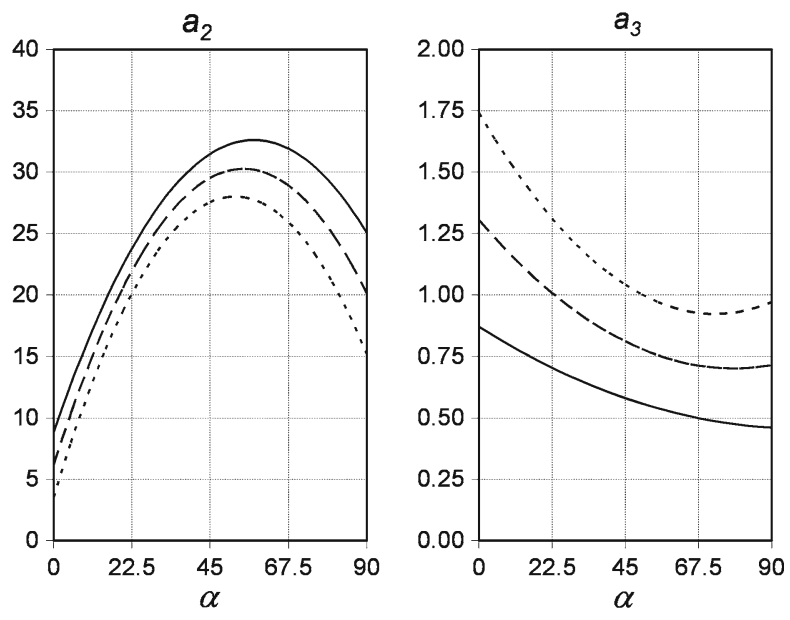

$-----W I H=2.0$

Fig. 11 Parameter $a_{1}, a_{2}$ and $a_{3}$ of Eq. 13 as functions of $W / H$ and $\alpha$ according to Eq. 14 
for smaller aspect ratios or two rows of avenues of trees for larger aspect ratios. For a perpendicular approaching flow, the estimate is pragmatic for configurations with $L / H>7$, since then the flow field in the inner canyon area is dominated by a canyon vortex (Hunter et al. 1991) with maximum pollutant concentrations at the leeward wall.

\section{Summary and Conclusions}

The impact of avenues of trees on traffic pollutant dispersion and concentrations in urban street canyons has been investigated. Avenues of trees of different tree-stand densities and crown porosities have been studied in street canyons with variations in the aspect ratio and wind direction.

Avenues of trees lead to alterations in the flow speeds and traffic pollutant concentration levels in comparison to the tree-free situations. For a perpendicular wind direction $\left(\alpha=90^{\circ}\right)$, with decreasing crown porosity or increasing tree-stand density, increases in wall-average and wall-maximum concentrations have been found at the leeward wall together with smaller decreases at the windward wall. For an oblique wind direction $\left(\alpha=45^{\circ}\right)$, increases in concentrations were observed at both canyon walls. Monotonic increases have been found for the wall-maximum concentrations with decreasing crown porosity or increasing tree-stand density. The wall-average concentrations were highest for intermediate crown porosities and tree-stand densities. For a parallel wind direction $\left(\alpha=0^{\circ}\right)$, steady increases in wall-average and wall-maximum concentrations have been observed with decreasing crown porosity or increasing tree-stand density.

The street-canyon aspect ratio affects the concentration level but not the characteristic concentration distribution at the canyon walls. Lower normalized pollutant concentrations are present in the broad street canyon. In general, the same trends in concentrations with crown porosity and tree-stand density have been identified in the narrow $(W / H=1)$ and broad street canyons $(W / H=2)$.

The study has revealed that the impacts of avenues of trees on traffic pollutant dispersion are greatest for an oblique wind direction $\left(\alpha=45^{\circ}\right)$, resulting in the largest concentrations. The largest changes referred to the tree-free situations have been found here with relative increases in the wall-average concentration of $146 \%$ and in the wall-maximum concentration of $205 \%$. Thus, the prevailing assumption, which attributes the most harmful dispersion conditions to a perpendicular wind, does not hold for street canyons with avenues of trees.

Following dimensional analytical considerations, a relationship for estimating the normalized wall-maximum traffic pollutant concentration $c_{\max }^{+}$has been derived. The wall-maximum concentration is governed by four parameters: (i) the approaching wind direction, (ii) the street-canyon aspect ratio, (iii) the crown porosity and (iv) the tree-stand density. The estimate can be applied in urban planning and design to assess the implications of avenues of trees on traffic pollutant concentrations in street canyons.

For future street-canyon dispersion studies we suggest investigating tree-avenues with high crown porosities, i.e. $P_{\mathrm{Vol}}>97.5 \%$ (corresponding to pressure loss coefficients $\lambda<80 \mathrm{~m}^{-1}$ ), since the measurement results indicate a strong sensitivity of the dispersion processes in the high porosity range. It is furthermore suggested studying in more detail wind directions in the sector between $30^{\circ}<\alpha<70^{\circ}$ as the wind-tunnel results suggest this range to be most critical for dispersion with the largest wall-maximum traffic pollutant concentrations. Also the influence of roof geometry on pollutant dispersion should be investigated in future studies. So far, the flat roof geometry has been studied exclusively for street canyons with avenues of trees. 
Acknowledgments The authors are indebted to the German Research Foundation DFG (Deutsche Forschungsgemeinschaft) for financial support (Grant No. Ru 345/28). The authors thank the anonymous reviewers for their valuable comments that helped to improve the manuscript and Christopher Hughes for the linguistic revision of the manuscript.

\section{References}

Ahmad K, Khare M, Chaudhry KK (2005) Wind tunnel simulation studies on dispersion at urban street canyons and intersections-a review. J Wind Eng Ind Aerodyn 93:697-717

Baik J, Kim J (1999) A numerical study of flow and pollutant dispersion characteristics in urban street canyons. J Appl Meteorol 38:1576-1589

Baik J, Kim J (2002) On the escape of pollutants from urban street canyons. Atmos Environ 36:527-536

Baik J, Park RS, Chun HY, Kim J (2000) A laboratory model of urban street-canyon flows. J Appl Meteorol 39:1592-1600

Baik J, Kang YS, Kim J (2007) Modeling reactive pollutant dispersion in an urban street canyon. Atmos Environ 41:934-949

Balczó M, Gromke C, Ruck B (2009) Numerical modeling of flow and pollutant dispersion in street canyons with tree planting. Meteorol Z 18:197-206

Blocken B, Stathopoulos T, Saathoff P, Wang X (2008) Numerical evaluation of pollutant dispersion in the built environment: comparisons between models and experiments. J Wind Eng Ind Aerodyn 96:18171831

Buccolieri R, Gromke C, Di Sabatino S, Ruck B (2009) Aerodynamic effects of trees on pollutant concentration in street canyons. Sci Total Environ 407:5247-5256

Buccolieri R, Salim SM, Leo LS, Di Sabatino S, Chan A, Ielpo P, de Gennaro G, Gromke C (2011) Analysis of local scale tree-atmosphere interaction on pollutant concentration in idealized street canyons and application to a real urban junction. Atmos Environ 45:1702-1713

CODASC (2008) Concentration Data of Street Canyons. Internet database. Karlsruhe Institute of Technology (KIT). www.codasc.de. Accessed 15 Jan 2012

DePaul FT, Sheih CM (1985) A tracer study of dispersion in an urban street canyon. Atmos Environ (1967) 19:555-559

DePaul FT, Sheih CM (1986) Measurements of wind velocities in a street canyon. Atmos Environ (1967) 20:455-459

Dezso-Weidinger G, Stitou A, van Beeck J, Riethmuller ML (2003) Measurement of the turbulent mass flux with PTV in a street canyon. J Wind Eng Ind Aerodyn 91:1117-1131

Eliasson I, Offerle B, Grimmond CSB, Lindqvist S (2006) Wind fields and turbulence statistics in an urban street canyon. Atmos Environ 40:1-16

Frank C, Ruck B (2005) Double-arranged mound-mounted shelterbelts: influence of porosity on wind reduction between the shelters. Environ Fluid Mech 5:267-292

Gandemer J (1981) The Aerodynamic characteristics of windbreaks, resulting in empirical design rules. J Wind Eng Ind Aerodyn 7:15-36

Grant PF, Nickling WG (1998) Direct field measurement of wind drag on vegetation for application of windbreak design and modeling. Land Degrad Dev 9:57-66

Gromke C (2008) Einfluss von Bäumen auf die Durchlüftung von innerstädtischen Straßenschluchten. Dissertation, Universitätsverlag Karlsruhe, Karlsruhe, 142 pp

Gromke C (2011) A vegetation modeling concept for Building and Environmental Aerodynamics wind tunnel tests and its application in pollutant dispersion studies. Environ Pollut 159:2094-2099

Gromke C, Ruck B (2005) Die Simulation atmosphärischer Grenzschichten in Windkanälen. In: Egbers C, Jehring L, Larcher T et al (eds) Lasermethoden in der Strömungsmesstechnik, vol 13. Cottbus, Germany, pp 51.1-51.8

Gromke C, Ruck B (2007) Influence of trees on the dispersion of pollutants in an urban street canyonExperimental investigation of the flow and concentration field. Atmos Environ 41:3287-3302

Gromke C, Ruck B (2008) Aerodynamic modeling of trees for small scale wind tunnel studies. Forestry $81: 243-258$

Gromke C, Ruck B (2009a) Effects of trees on the dilution of vehicle exhaust emissions in urban street canyons. Int J Environ Waste Manag 4:225-242

Gromke C, Ruck B (2009b) On the impact of trees on dispersion processes of traffic emissions in street canyons. Boundary-Layer Meteorol 131:19-34

Gromke C, Buccolieri R, Di Sabatino S, Ruck B (2008) Dispersion modeling study in a street canyon with tree planting by means of wind tunnel and numerical investigations - Evaluation of CFD data with experimental data. Atmos Environ 42:8640-8650 
Gross G (1987) A numerical study of the air flow within and around a single tree. Boundary-Layer Meteorol 40:311-327

Gross G (1997) ASMUS - Ein numerisches Modell zur Berechnung der Strömung und der Schadstoffverteilung im Bereich einzelner Gebäude. Teil II: Schadstoffausbreitung und Anwendung. Meteorol Z 6:130-136

Grunert F, Benndorf D, Klingbeil K (1984) Neuere Ergebnisse zum Aufbau von Schutzpflanzungen. Beiträge für die Forstwissenschaft 18:108-115

Huang Y, Hu X, Zeng N (2009) Impact of wedge-shaped roofs on airflow and pollutant dispersion inside urban street canyons. Build Environ 44:2335-2347

Hunter LJ, Watson ID, Johnson GT (1991) Modelling air flow regimes in urban canyons. Energy Build 15: 315-324

Kastner-Klein P, Plate EJ (1999) Wind-tunnel study of concentration fields in street canyons. Atmos Environ 33:3973-3979

Kastner-Klein P, Fedorovich E, Rotach MW (2001) A wind tunnel study of organised and turbulent air motions in urban street canyons. J Wind Eng Ind Aerodyn 89:849-861

Langner M, Kull M, Endlicher WR (2011) Determination of PM10 deposition based on antimony flux to selected urban surfaces. Environ Pollut 159:2028-2034

Li X, Liu C, Leung DYC, Lam KM (2006) Recent progress in CFD modelling of wind field and pollutant transport in street canyons. Atmos Environ 40:5640-5658

Litschke T, Kuttler W (2008) On the reduction of urban particle concentration by vegetation-a review. Meteorol Z 17:229-249

Liu C, Barth MC (2002) Large-eddy simulation of flow and scalar transport in a modeled street canyon. J Appl Meteorol 41:660-673

Meroney RN (2004) Wind tunnel and numerical simulation of pollution dispersion: a hybrid approach. Working Paper. Croucher Advanced Study Institute on Wind Tunnel Modeling, Hong Kong University of Science and Technology. http://www.engr.colostate.edu/ meroney/projects/ASI\%20Crocher\% 20Paper\%20Final.pdf. Accessed 15 Jan 2012

Meroney RN, Pavageau M, Rafailidis S et al (1996) Study of line source characteristics for 2-D physical modelling of pollutant dispersion in street canyons. J Wind Eng Ind Aerodyn 62:37-56

Moonen P, Dorer V, Carmeliet J (2011a) Evaluation of the ventilation potential of courtyards and urban street canyons using RANS and LES. J Wind Eng Ind Aerodyn 99:414-423

Moonen P, Gromke C, Dorer V, Carmeliet J (2011b) LES of dispersion in a street canyon with tree planting. In: Leitl B (ed) Physical modelling of flow and dispersion phenomena PHYSMOD2011, Hamburg Germany, pp 320-327

Pavageau M, Schatzmann M (1999) Wind tunnel measurements of concentration fluctuations in an urban street canyon. Atmos Environ 33:3961-3971

Ries K, Eichhorn J (2001) Simulation of effects of vegetation on the dispersion of pollutants in street canyons. Meteorol Z 10:229-233

Ruck B, Schmidt F (1986) Das Strömungsfeld der Einzelbaumumströmung. Forstwiss Centralblatt 105: 178-196

Salim SM, Cheah SC, Chan A (2011) Numerical simulation of dispersion in urban street canyons with avenue-like tree plantings: Comparison between RANS and LES. Build Environ 46:1735-1746

Schatzmann M, Leitl B (2002) Validation and application of obstacle-resolving urban dispersion models. Atmos Environ 36:4811-4821

Schlichting H, Gersten K (2003) Boundary layer theory. Spriner, Berlin, 801 pp

Sini JF, Anquetin S, Mestayer PG (1996) Pollutant dispersion and thermal effects in urban street canyons. Atmos Environ 30:2659-2677

Snyder WH (1972) Similarity criteria for the application of fluid models to the study of air pollution meteorology. Boundary-Layer Meteorol 3:113-134

So ESP, Chan ATY, Wong AYT (2005) Large-eddy simulations of wind flow and pollutant dispersion in a street canyon. Atmos Environ 39:3573-3582

Soulhac L, Perkins RJ, Salizzoni P (2008) Flow in a street canyon for any external wind direction. BoundaryLayer Meteorol 126:365-388

Stull RB (1988) An introduction to boundary layer meteorology. Springer, Berlin, 670 pp

Vardoulakis S, Fisher BEA, Pericleous K, Gonzalez-Flesca N (2003) Modelling air quality in street canyons: a review. Atmos Environ 37:155-182

VDI 3783-12 (2000) Environmental meteorology: physical modelling of flow and dispersion processes in the atmospheric boundary layer-application of wind tunnels. Verein Deutscher Ingenieure (ed) Beuth Verlag, Berlin, 36 pp 